\title{
RIQUEZA E COMPOSIÇÃO DE ESPÉCIES DE INSETOS VISITANTES FLORAIS DE ALGODOEIRO BT E NÃO-BT*
}

\author{
C.C. Dutra**, C. Meotti***, M.G. Fernandes, J. Raizer
}

Universidade Federal da Grande Dourados, Rod. Dourados-Itahum, km 12, CEP 79804-970, Dourados, MS, Brasil. E-mail: carlacristina.dutra@gmail.com

\section{RESUMO}

O objetivo deste estudo foi verificar se existe diferença entre a comunidade de insetos visitantes florais do algodoeiro geneticamente modificado (Bt) e sua isolinha convencional (não-Bt). A pesquisa foi realizada em área de produção comercial no Município de Maracaju, MS, Brasil. A área amostral compreendeu dois campos, um de algodão não-Bt e outro de algodão Bt. As coletas dos insetos foram realizadas no período matutino com intervalo de três dias. Ao final da floração foram coletados 1.310 espécimes de visitantes florais, destes, 56,56\% nas flores do algodoeiro Bt e 43,44\% do não-Bt. Embora a abundância de insetos no cultivar Bt ter sido maior, a riqueza de espécies no cultivar não-Bt foi superior. Assim, apresentando diversidade de 73 espécies na cultivar Bt e 84 espécies na não-Bt. A riqueza de espécies variou entre os horários de coleta para ambos os tratamentos, entre os dias de floração onde no início e no final da floração, constatou-se a menor riqueza independentemente do cultivar de algodão. Por outro lado, a riqueza de espécies não variou entre os cultivares de algodão. Com relação à composição das espécies houve variação entre os horários de visitação, entre os tipos de algodão e também entre a interação, desses dois fatores. Também variou entre os dias de floração, mas não entre as classes da interação os dias e o tipo de algodão. Com base neste estudo, concluímos que houve diferença entre as espécies de insetos que visitaram as flores do algodoeiro Bt e sua isolinha convencional.

PALAVRAS-CHAVE: Algodão, análise derisco, Bacillus thuringiensis, plantas geneticamente modificas.

\section{ABSTRACT}

RICHNESS AND DIVERSITY OF FLOWER-VISITING INSECT SPECIES IN BT AND NON-BT COTTON. This study aimed to verify the difference between flower-visiting insect communities in genetically modified cotton $(\mathrm{Bt})$ and conventional cotton (non-Bt). The study was conducted in a commercial cotton field in the municipality of Maracaju, Mato Grosso doSul, Brazil. The collections of flower visitors were performed every 3 days in the morning during the reproductive period of cotton and employed 2 treatments: Bt cotton and non-Bt cotton. By the end of flowering, 1,310 specimens were collected, $56.56 \%$ on Bt cotton flowers and $43.44 \%$ on non-Bt cotton flowers. Even though the abundance of collected insects on Bt cotton was higher, species richness in non-Bt was higher than Bt, with a diversity of 73 species on Bt cotton and 84 species on non-Bt. Species richness of flower visitors varied between sampling times for both treatments and between the days of flowering, therefore, the species richness was lower at the beginning and the end of flowering in both cotton cultivars. But the species richness did not vary between cultivars of cotton. Species composition of insects was different during the periods of flower visitation, between cotton cultivars as well as between the interaction of these two factors. There was also a variation between the days of flowering but not between days and cotton cultivars. Based on this study, it was concluded that there are differences between the species of insects that visit flowers of Bt cotton and non-Bt cotton.

KEY WORDS: Cotton, risk assessment, Bacillus thuringiensis, genetically modified plants.

\section{INTRODUÇÃO}

Asplantasgeneticamentemodificadas(GM)queexpressamendotoxinas provenientes da bactéria Bacillus thuringiensis Berliner, atuam como bioinseticidas e são importantes ferramentas para auxiliar no manejo integrado de pragas agrícolas. Os EUA ocupam a primeira posição no quadro mundial em relação à

*Apoio: Capes.

**Programa de Pós-Graduação em Agronomia, UFGD.

***Programa de Pós-Graduação em Entomologia e Conservação da Biodiversidade, UFGD. 
área plantada com cultivares transgênicos e o Brasil ocupa a segunda posição (INTERNATIONAL ..., 2009).

Das variedades GM para controle de insetos plantadas no Brasil destacam-se as de algodão e de milho. $\mathrm{O}$ algodão Bollgard ${ }^{\circledR}$ (MON531) foi a primeira variedade aprovada pela Comissão Técnica de Biossegurança no Brasil em 2005 (Comissão..., 2008). Essa variedade possui um gene (genes Cry) proveniente da Bacillus thuringiensis (Bt) que codifica a proteína Cry1Ac, a qual tem como alvo insetos da ordem Lepidoptera: Alabama argillacea (Hüeber), Heliothis virescens (Fabricius) e Pectinophora gossypiella (Saunders). Posteriormente, em 2009, foram aprovadas outras variedades de algodão GM que expressam outras proteínas Cry resistentes a outros lepidópteros pragas (ComISsão..., 2009). Sendo que essas concentrações de toxinas Bt nos tecidos de pólen, folha, raiz e sementes são variáveis de acordo com os diferentes genes que codificam proteínas de ação inseticida inseridos na planta (ANDOW, 2008).

$\mathrm{Na}$ cultura do algodão no Brasil ocorrem vários períodos de alta infestação com lagartas, os quais resultam em redução no rendimento, aumento das aplicações de inseticidas, causando poluição ambiental e elevando o custo de produção. Nesse sentido, a tecnologia Bt seinsere como alternativa à utilização de inseticidas químicos, desde que os riscos ambientais resultantes de sua utilização sejam mínimos ou inexistentes. Omanejo de pragas com base em cultivos Bt tem várias vantagens significativas sobre inseticidas sintéticos tradicionais, pois a toxina se acumula de forma homogênea nos tecidos vegetais das plantas e exibe um alto grau de especificidade para os insetosalvo da tecnologia, devendo ser ingerida para exercer seus efeitos, uma vez que não possui atividade por contato (BETz et al., 2000; ANDOW, 2008). Entretanto, existem evidências de que a expressão de toxinas $\mathrm{Bt}$, na planta transgênica, pode alterar (direta ou indiretamente) as populações de espécies não-alvo (BIRCH, 1997; MonNERAT; Bravo, 2000). Tal como a redução de populações de insetos polinizadores e visitantes florais em geral como besouros e borboletas, predadores e parasitoides, o que pode levar a redução do controle biológico de pragas (Hong et al., 2008). A preservação e manutenção dos inimigos naturais são imprescindíveis para estabelecer o equilíbrio biológico e reduzir os custos de produção (GRAVENA, 1983). Com base nisto, o objetivo deste estudo foi verificar se existe diferença entre a comunidade de insetos visitantes florais do algodoeiro GM (Bt) e sua isolinha convencional (não-Bt).

\section{MATERIAL E MÉTODOS}

Área experimental.Oexperimento foi conduzido na Fazenda Comporta, no Município de Maracaju,

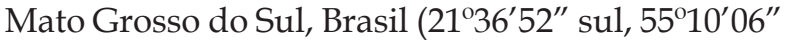
oestee altitude de $384 \mathrm{~m}$ ). Aárea amostral compreendeu dois campos de $100 \mathrm{~m}$ por 18 linhas cada um, instalados em uma lavoura comercial de algodão Gossypium hirsutum L. Um campo foi semeado com o cultivar convencional DeltaOpal ${ }^{\circledR}$ e o outro com seu cultivar isolinha GM resistente a insetos $\mathrm{NuOpal}^{\circledR}$ Bollgard $^{\circledR}$ (MON531). A área amostral foi isenta de aplicações de inseticidas e, para o manejo de plantas daninhas, foram realizadas duas capinas manual.

A semeadura de ambos os cultivares de algodão foi realizada no dia 15 de novembro de 2007, e as coletas dos visitantes florais ocorreram durante todo o período de floração do algodoeiro (8 de janeiro a 14 de fevereiro de 2008). Cada campo foi dividido em três parcelas de $100 \mathrm{~m}$ de comprimento por seis linhas de largura. Entre os campos foi delimitada uma área de nove metros para dar um intervalo entre as duas variedades, na dimensão de $100 \mathrm{~m}$ de comprimento por 10 linhas de largura.

Levantamento dos visitantes florais. $\mathrm{O}$ método de amostragem foi adaptado de PIREs et al. (2006a). Coletas dos indivíduos visitantes florais foram feitas buscando-se determinar a abundância ea diversidade desses insetos no algodão Bt e não-Bt, assim como o horário de visitação e a composição de espécies em cada horário avaliado em ambos os cultivares.

As coletas foram feitas com intervalo de três dias, durante todo período de floração da cultura. Totalizando nove dias de amostragem com seis coletas diárias (das $7 \mathrm{~h}$ às $12 \mathrm{~h}$ ). Que consistiu de 54 repetições em cada parcela, totalizando 324 coletas (162 em cada tratamento). O esforço de coleta foi de 15 minutos percorridos por cada coletor ao longo dos $100 \mathrm{~m}$ de sua parcela.

A coleta foi manual, com o auxílio de rede entomológica, ou diretamente no frasco mortífero. Todos os insetos encontrados nas flores foram capturados. Após os 15 minutos, os insetos, já mortos, foram colocados em um envelope, constando data, hora, coletor e parcela de coleta e, posteriormente, armazenados em caixas de isopor, levados para o Laboratório de Biologia da Universidade Federal da Grande Dourados (UFGD), para posterior triagem.

Identificação dos visitantes florais. Os insetos foram identificados no nível específico por especialistas da Universidade Federal do Paraná (UFPR), onde foram feitas as montagens e identificação do material no menor nível taxonômico possível.

Análise estatística. Para analisar a diversidade das duas comunidades estudadas, foi calculada a função Shannon-Wiener (SHANNON; WEAVER, 1949). Visando a obter gradientes representativos da estrutura da comunidade de visitantes florais, baseada na composição de espécies, em cada horário e dia de coleta, utilizou-se o método ordenação de escalona- 
mento multidimensional híbrido (HMDS) (FAITH et al., 1987), com índice de dissimilaridade Bray-curtis (BRAY; CURTIS, 1957) como medida de associação entre os possíveis pares de amostras. Foram consideradas as abundâncias relativas das 34 espécies que foram coletadas em, no mínimo, três amostras. O número de dimensões que melhor representou a ordenação foi decidido pela comparação dos valores de ajuste $\left(\mathrm{r}^{2}\right)$ obtidos na regressão linear entre os valores das distâncias matrizes de Bray-curtis e dos valores derivados da ordenação para uma, duas ou três dimensões.

\section{RESULTADOS E DISCUSSÃO}

Durante o período de florescimento dos cultivares, foram coletados 1.310 espécimes de visitantes florais. Destes, 56,56\% (741) estavam presentes nas flores do algodoeiro Bt, e 43,44\% (569) do algodoeiro não-Bt (Tabela 1). Embora a abundância de insetos coletados no cultivar Bt ter sido maior, a diversidade de espécies no cultivar não-Bt foi superior. Destarte, apresentando riqueza de 73 espécies de visitantes florais no cultivar Bt e 84 espécies no cultivar não-Bt.

Os coleópteros da família Coccinellidae foram mais abundantes no cultivar Bt (254 espécimes) do que no não-Bt (115 espécimes) (Tabela 1), o que pode estar relacionado com a presença de presa como o pulgão-do-algodoeiro (Aphis gossypii). Além de serem insetos predadores, também são considerados visitantes florais do algodoeiro, pois se alimentam de recursos florais como o pólen (ARPAIA et al., 2006; Hong et al., 2008).

Dos insetos encontrados nas flores do algodão $\mathrm{Bt}$, a maior riqueza foi representada pela ordem Hymenoptera com um total de 24 espécies, posteriormente Diptera com 22 espécies, Coleoptera com 14 espécies, Lepidoptera com 12 espécies e Dermaptera com apenas uma espécie. No algodão não-Bt, a maior riqueza de espécies também foi da ordem Hymenoptera com 32 espécies, seguida de Diptera com 27 espécies, Coleoptera com 15 espécies e Lepidoptera com 10 espécies.

Dentre os himenópteros coletados, aqueles da família Apoidea foram os mais abundantes, representando $24,50 \%$ dos insetos encontrados, totalizando 13 espécies de abelhas em ambos os cultivares, sendo que Apis mellifera foi a espécie mais abundante (299 espécimes), PIREs et al. (2006b) também obteve resultados parecidos em outras regiões do Brasil (Bahia, Goiás, Mato Grosso, São Paulo e no Distrito Federal) assim como Telles (2006) em Rondonópolis, MT. Muitos estudos conduzidos em algodoeiro confirmam que as abelhas são o principal grupo de polinizadores em todas as regiões brasileiras (ANACLETO; MARCHINI, 2005; SANCHEZ-Junior; Malerbo-SOUZA, 2004; Thapa, 2006). Contudo, a diversidade de espécies de abelha associada ao Gossypium spp. deve ser estudada por região geográfica, o que permite a afirmação de análise de risco de plantas geneticamente modi-

Tabela 1 - Número de indivíduos (N) e índice de diversidade de Shannon-Wiener $(\mathrm{H})$ das espécies de visitantes florais, registrados em cultivares de algodão convencional (não-Bt) e GM (Bt). Maracaju-MS, Brasil, 2008.

\begin{tabular}{|c|c|c|c|c|c|}
\hline \multirow{2}{*}{ Táxon } & \multirow{2}{*}{ Espécie } & \multicolumn{2}{|r|}{$\mathrm{Bt}$} & \multicolumn{2}{|c|}{$\mathrm{N}-\mathrm{Bt}$} \\
\hline & & $\mathrm{N}$ & $\mathrm{H}$ & $\mathrm{N}$ & $\mathrm{H}$ \\
\hline \multicolumn{6}{|l|}{ Hymenoptera } \\
\hline \multirow[t]{9}{*}{ Apidae } & Tetragonisca angustula (Latreille,1807) & 0 & 0 & 2 & $-0,01986$ \\
\hline & Geotrigona sp.1 (Moure, 1943) & 0 & & 2 & \\
\hline & Trigona hyalinata (Lepeletier,1836) & 0 & 0 & 1 & $-0,01115$ \\
\hline & Trigona spinipes (Fabricius, 1793) & 2 & $-0,01596$ & 2 & $-0,01986$ \\
\hline & Bombus (Fervidobombus) morio (Swederus, 1787) & 2 & $-0,01596$ & 2 & $-0,01986$ \\
\hline & Xylocopa (Neoxylocopa) brasilianorum (Linnaeus,1767) & 1 & $-0,00892$ & 0 & 0 \\
\hline & Exomalopsis (Exomalopsis) analis (Spinola,1853) & 1 & $-0,00892$ & 0 & 0 \\
\hline & Apis mellifera (Linnaeus,1758) & 160 & $-0,33097$ & 139 & $-0,3443$ \\
\hline & Eulaema (Apeulaema) nigrita (Lepeletier,1841) & 0 & 0 & 1 & $-0,01115$ \\
\hline Andrenidae & Oxaea flavescens (Klug,1807) & 1 & $-0,00892$ & 2 & $-0,01986$ \\
\hline \multirow[t]{3}{*}{ Halictidae } & Augochloropsis cleopatra (Schrottky,1902) & 1 & $-0,00892$ & 0 & 0 \\
\hline & Halictus sp. (Latreille, 1804) & 0 & 0 & 1 & $-0,01115$ \\
\hline & Paraxystoglossa cf. jocasta (Schrottky,1910) & 0 & 0 & 1 & $-0,01115$ \\
\hline \multirow[t]{6}{*}{ Formicidae } & Formicidae Gênero A sp.1 & 1 & $-0,00892$ & 0 & 0 \\
\hline & Brachymyrmex sp. (Mayr,1868) & 1 & $-0,00892$ & 0 & 0 \\
\hline & Pheidole sp.3 (Westwood,1840) & 1 & $-0,00892$ & 0 & 0 \\
\hline & Dorymyrmex sp. (Mayr,1866) & 1 & $-0,00892$ & 0 & 0 \\
\hline & Pheidole sp.1 (Westwood,1840) & 1 & $-0,00892$ & 0 & 0 \\
\hline & Solenopsis sp. (Westwood,1840) & 10 & $-0,0581$ & 0 & 0 \\
\hline
\end{tabular}

Continua. 
Tabela 1 - Continuação.

\begin{tabular}{|c|c|c|c|c|c|}
\hline \multirow{2}{*}{ Táxon } & \multirow{2}{*}{ Espécie } & \multicolumn{2}{|r|}{$\mathrm{Bt}$} & \multicolumn{2}{|c|}{$\mathrm{N}-\mathrm{Bt}$} \\
\hline & & $\mathrm{N}$ & $\mathrm{H}$ & $\mathrm{N}$ & $\mathrm{H}$ \\
\hline \multirow[t]{7}{*}{ Vespidae } & Stenodynerus sp. (de Saussure, 1863) & 0 & 0 & 1 & $-0,01115$ \\
\hline & Pachodynerus guadalupensis (de Saussure,1853) & 0 & 0 & 1 & $-0,01115$ \\
\hline & Pachodynerus serrulatus (Brèthes) & 0 & 0 & 1 & $-0,01115$ \\
\hline & Protonectarina sylveirae (de Saussure,1854) & 0 & 0 & 1 & $-0,01115$ \\
\hline & Polybia (Myrapetra) paulista (Ihering,1896) & 1 & $-0,00892$ & 1 & $-0,01115$ \\
\hline & Brachygastra lecheguana (Latreille,1824) & 1 & $-0,00892$ & 1 & $-0,01115$ \\
\hline & Agelaia pallipes (Olivier, 1791) & 0 & 0 & 1 & $-0,01115$ \\
\hline \multirow[t]{3}{*}{ Pompilidae } & Chalcochares sp. (Banks,1917) & 0 & 0 & 2 & $-0,01986$ \\
\hline & Aplochares sp. (Banks,1944) & 1 & $-0,00892$ & 1 & $-0,01115$ \\
\hline & Agenioideus sp. (Ashmead, 1902) & 0 & 0 & 1 & $-0,01115$ \\
\hline \multirow[t]{3}{*}{ Tiphiidae } & Anthoboscinae Gênero A sp.1 & 0 & 0 & 1 & $-0,01115$ \\
\hline & Anthoboscinae Gênero B sp.1 & 0 & 0 & 1 & $-0,01115$ \\
\hline & Anthoboscinae Gênero C sp.1 & 0 & 0 & 1 & $-0,01115$ \\
\hline Sphecidae & Ammophila sp.1 (Kirby, 1798) & 0 & 0 & 2 & $-0,01986$ \\
\hline Crabronidae & Liris sp. (Fabricius, 1804) & 0 & 0 & 1 & $-0,01115$ \\
\hline Trigonalyidae & Trygonalyidae Gênero A sp.1 & 0 & 0 & 1 & $-0,01115$ \\
\hline Chalcididae & Chalcididae Gênero A sp.1 & 0 & 0 & 1 & $-0,01115$ \\
\hline Pteromalidae & Pteromalidae Gênero A sp.1 & 0 & 0 & 1 & $-0,01115$ \\
\hline \multirow[t]{4}{*}{ Ichneumonidae } & Ichneumonidae Gênero A sp.1 & 7 & $-0,04404$ & 23 & $-0,12969$ \\
\hline & Ichneumonidae Gênero B sp.1 & 1 & $-0,00892$ & 0 & 0 \\
\hline & Ichneumonidae Gênero C sp.1 & 1 & $-0,00892$ & 0 & 0 \\
\hline & Ichneumonidae Gênero D sp.1 & 0 & 0 & 1 & $-0,01115$ \\
\hline Braconidae & Braconidae Gênero A sp.1 & 0 & 0 & 1 & $-0,01115$ \\
\hline \multicolumn{2}{|c|}{ Total de indivíduos da ordem Hymenoptera } & 195 & & 198 & \\
\hline \multicolumn{2}{|c|}{ Número de espécies da ordem Hymenoptera } & 24 & & 32 & \\
\hline \multicolumn{6}{|c|}{ Coleoptera } \\
\hline \multirow[t]{5}{*}{ Coccinellidae } & Scymnus (scymnus) sp. (Kugelann,1794) & 3 & $-0,02231$ & 12 & $-0,08139$ \\
\hline & Cycloneda sanguinea (Linnaeus,1763) & 64 & $-0,21153$ & 12 & $-0,08139$ \\
\hline & Eriopis connexa (Germar,1824) & 33 & $-0,13857$ & 6 & $-0,048$ \\
\hline & Hippodamia convergens (Guérin-Méneville,1842) & 152 & $-0,32495$ & 84 & $-0,28242$ \\
\hline & Hyperaspis festiva (Mulsant,1850) & 2 & $-0,01596$ & 1 & $-0,01115$ \\
\hline \multirow[t]{3}{*}{ Chrysomelidae } & Diabrotica sp. (Chevrolat, 1837) & 45 & $-0,17012$ & 14 & $-0,09116$ \\
\hline & Colaspis sp. (Fabricius, 1801) & 1 & $-0,00892$ & 0 & 0 \\
\hline & Colaspis joliveti (Bechyné,1955) & 29 & $-0,12683$ & 10 & $-0,07103$ \\
\hline \multirow[t]{3}{*}{ Elateridae } & Conoderus malleatus (Germar, 1824) & 9 & $-0,05357$ & 10 & $-0,07103$ \\
\hline & Elaterinae sp.1 & 0 & 0 & 1 & $-0,01115$ \\
\hline & Elaterinae sp.2 & 1 & $-0,00892$ & 1 & $-0,01115$ \\
\hline Carabidae & Odontochila sp. (Agassiz, 1847) & 1 & $-0,00892$ & 2 & $-0,01986$ \\
\hline \multirow[t]{2}{*}{ Scarabaeidae } & Macrodactylus sp. (Dejean,1821) & 4 & $-0,02819$ & 4 & $-0,03485$ \\
\hline & Euphoria lurida (Fabricius,1775) & 0 & 0 & 2 & $-0,01986$ \\
\hline \multirow[t]{2}{*}{ Curculionidae } & Anthonomus grandis (Boheman,1843) & 7 & $-0,04404$ & 0 & 0 \\
\hline & Sternechus subsignatus (Boheman,1836) & 1 & $-0,00892$ & 1 & $-0,01115$ \\
\hline Tenebrionidae & Tenebrionidae sp.1 & 0 & 0 & 1 & $-0,01115$ \\
\hline \multicolumn{2}{|c|}{ Total de indivíduos da ordem Coleoptera } & 352 & & 161 & \\
\hline Número de espé & ies da ordem Coleoptera & 14 & & 15 & \\
\hline Lepidoptera & & & & & \\
\hline Hesperiidae & Pyrgus oileus orcus (Stöll, 1780) & 1 & $-0,00892$ & 0 & 0 \\
\hline & Heliopetes omrina (Butler,1870) & 0 & 0 & 1 & $-0,01115$ \\
\hline & Calpodes ethlius (Stoll,1782) & 1 & $-0,00892$ & 0 & 0 \\
\hline & Nyctelius nyctelius nyctelius (Latreille, 1824) & 1 & $-0,00892$ & 0 & 0 \\
\hline Sphyngidae & Erinnyis ello (Linnaeus,1758) & 4 & $-0,02819$ & 3 & $-0,02765$ \\
\hline Pieridae & Glutophrissa drusilla drusilla (Cramer, 1777) & 1 & $-0,00892$ & 0 & 0 \\
\hline Nymphalidae & Heliconius erato phyllis (Fabricius,1775) & 0 & 0 & 1 & $-0,01115$ \\
\hline Noctuidae & Anticarsia gemmatalis (Hubner,1818) & 32 & $-0,1357$ & 19 & $-0,11351$ \\
\hline & Heliothis virescens (Fabricius,1781) & 20 & $-0,0975$ & 43 & $-0,19518$ \\
\hline & Pseudoplusia includes (Walker,1857) & 56 & $-0,19518$ & 35 & $-0,17153$ \\
\hline
\end{tabular}

Continua. 
Tabela 1 - Continuação.

\begin{tabular}{|c|c|c|c|c|c|}
\hline \multirow{2}{*}{ Táxon } & \multirow{2}{*}{ Espécie } & \multicolumn{2}{|r|}{$\mathrm{Bt}$} & \multicolumn{2}{|c|}{$\mathrm{N}-\mathrm{Bt}$} \\
\hline & & $\mathrm{N}$ & $\mathrm{H}$ & $\mathrm{N}$ & $\mathrm{H}$ \\
\hline & Alabama argilacea (Hubner,1818) & 4 & $-0,02819$ & 13 & $-0,08634$ \\
\hline & Spodoptera frugiperda (J. E. Smith, 1797) & 2 & $-0,01596$ & 2 & $-0,01986$ \\
\hline & Elaphria agrotina (Guenée,1852) & 2 & $-0,01596$ & 3 & $-0,02765$ \\
\hline Pyralidae & Pyralidae sp. & 10 & $-0,0581$ & 4 & $-0,03485$ \\
\hline \multicolumn{2}{|c|}{ Total de indivíduos da ordem Lepidoptera } & 134 & & 124 & \\
\hline \multicolumn{2}{|c|}{ Número de espécies da ordem Lepidoptera } & 12 & & 10 & \\
\hline \multicolumn{6}{|c|}{ Diptera } \\
\hline \multirow[t]{9}{*}{ Syrphidae } & Allograpta obliqua (Say,1823) & 2 & $-0,01596$ & 0 & 0 \\
\hline & Allograpta exótica (Wiedemann,1830) & 26 & $-0,11754$ & 46 & $-0,20334$ \\
\hline & Ocyptamus sp. (Macquart, 1834) & 1 & $-0,00892$ & 1 & $-0,01115$ \\
\hline & Ornidia obesa (Lepeletier \& Serville, 1828) & 0 & 0 & 1 & $-0,01115$ \\
\hline & Pseudodorus clavatus (Fabricius,1794) & 0 & 0 & 1 & $-0,01115$ \\
\hline & Palpada sp. (Macquart, 1834) & 0 & 0 & 1 & $-0,01115$ \\
\hline & Ocyptamus gastrostactus (Wiedmann, 1830) & 1 & $-0,00892$ & 3 & $-0,02765$ \\
\hline & Ocyptamus antiphates (Walker, 1849) & 0 & 0 & 3 & $-0,02765$ \\
\hline & Toxomorus politus (Say,1823) & 0 & 0 & 1 & $-0,01115$ \\
\hline \multirow[t]{3}{*}{ Asilidae } & Asilidae Gênero A sp.1 & 1 & $-0,00892$ & 0 & 0 \\
\hline & Asilidae Gênero B sp.1 & 1 & $-0,00892$ & 0 & 0 \\
\hline & Asilidae Gênero C sp.1 & 0 & 0 & 2 & $-0,01986$ \\
\hline Bombylidae & Bombylidae sp. & 3 & $-0,02231$ & 4 & $-0,03485$ \\
\hline Calliphoridae & Calliphoridae sp. & 1 & $-0,00892$ & 0 & 0 \\
\hline Cullicidae & Culicidae sp. & 1 & $-0,00892$ & 0 & 0 \\
\hline Stratiomidae & Stratiomidae sp. & 1 & $-0,00892$ & 2 & $-0,01986$ \\
\hline Dolichopodidae & Dolichopodidae sp. & 1 & $-0,00892$ & 0 & 0 \\
\hline \multirow[t]{20}{*}{ Tachinidae } & Tachinidae sp.1 & 4 & $-0,02819$ & 3 & $-0,02765$ \\
\hline & Tachinidae sp.2 & 3 & $-0,02231$ & 1 & $-0,01115$ \\
\hline & Tachinidae sp.3 & 0 & 0 & 3 & $-0,02765$ \\
\hline & Tachinidae sp.4 & 4 & $-0,02819$ & 3 & $-0,02765$ \\
\hline & Tachinidae sp.5 & 1 & $-0,00892$ & 1 & $-0,01115$ \\
\hline & Tachinidae sp.6 & 0 & 0 & 1 & $-0,01115$ \\
\hline & Tachinidae sp.7 & 0 & 0 & 1 & $-0,01115$ \\
\hline & Tachinidae sp.8 & 0 & 0 & 1 & $-0,01115$ \\
\hline & Tachinidae sp.9 & 0 & 0 & 1 & $-0,01115$ \\
\hline & Tachinidae sp.10 & 0 & 0 & 1 & $-0,01115$ \\
\hline & Tachinidae sp.11 & 1 & $-0,00892$ & 0 & 0 \\
\hline & Tachinidae sp.12 & 1 & $-0,00892$ & 0 & 0 \\
\hline & Tachinidae sp.13 & 0 & 0 & 1 & $-0,01115$ \\
\hline & Tachinidae sp.14 & 1 & $-0,00892$ & 0 & 0 \\
\hline & Tachinidae sp.15 & 0 & 0 & 1 & $-0,01115$ \\
\hline & Tachinidae sp.16 & 1 & $-0,00892$ & 0 & 0 \\
\hline & Tachinidae sp.17 & 1 & $-0,00892$ & 0 & 0 \\
\hline & Tachinidae sp.18 & 0 & 0 & 1 & $-0,01115$ \\
\hline & Tachinidae sp.19 & 0 & 0 & 1 & $-0,01115$ \\
\hline & Tachinidae sp.20 & 0 & 0 & 1 & $-0,01115$ \\
\hline \multicolumn{2}{|c|}{ Total de indivíduos da ordem Diptera } & 56 & & 86 & \\
\hline \multirow{2}{*}{\multicolumn{2}{|c|}{$\begin{array}{l}\text { Número de espécies da ordem Diptera } \\
\text { Dermaptera }\end{array}$}} & 22 & & 27 & \\
\hline & & & & & \\
\hline Forficulidae & Doru lineare (Esch,1822) & 4 & $-0,02819$ & 0 & 0 \\
\hline \multicolumn{2}{|c|}{ Total de indivíduos da ordem Dermaptera } & 4 & & 0 & \\
\hline \multicolumn{2}{|c|}{ Número de espécies da ordem Dermaptera } & 1 & & 0 & \\
\hline \multicolumn{2}{|l|}{ Total } & 741 & 2,759711 & 569 & 3,019509 \\
\hline
\end{tabular}

ficadas no Brasil (Pires et al., 2006b).

Os parasitoides da família Ichneumonidae foram encontrados com frequência no algodão não-Bt, representando $2,51 \%$ dos insetos coletados nos dois cultivares de algodão, e espécies de Sphecidae e Pompilidae representaram $0,50 \%$ do total dos himenópteros coletados. Já entre os dípteros, 6,59\% do total de insetos coletados pertencem à família 
Syrphidae e, destes 60,38\% estavam em flores do algodão Bt (Tabela 1). Número de indivíduos (N) e índice de diversidade de Shannon-Wiener $(\mathrm{H})$ das espécies de visitantes florais, registrados em cultivares de algodão convencional (não-Bt) e GM (Bt). Maracaju, MS, Brasil, 2008.

Com relação ao horário de visitação, énecessário ressaltar que houve diferença entre a riqueza de espécies no decorrer da manhã para os dois tipos de algodão $\left(\mathrm{r}^{2}=0,925 ; \mathrm{F}=56,76 ; \mathrm{gl}=5 ; \mathrm{p}<0,001\right)$ (Fig. 1). O horário que houve maior incidência de espécies foi das $9 \mathrm{~h}$ às $11 \mathrm{~h}$ da manhã (Tabela 2), e o menor número de espécies foi entre $12 \mathrm{~h}$ e $13 \mathrm{~h}$. $O$ pico de insetos visitantes às $9 \mathrm{~h}$ pode ser devido a maior produção e oferecimento de recursos para os insetos.

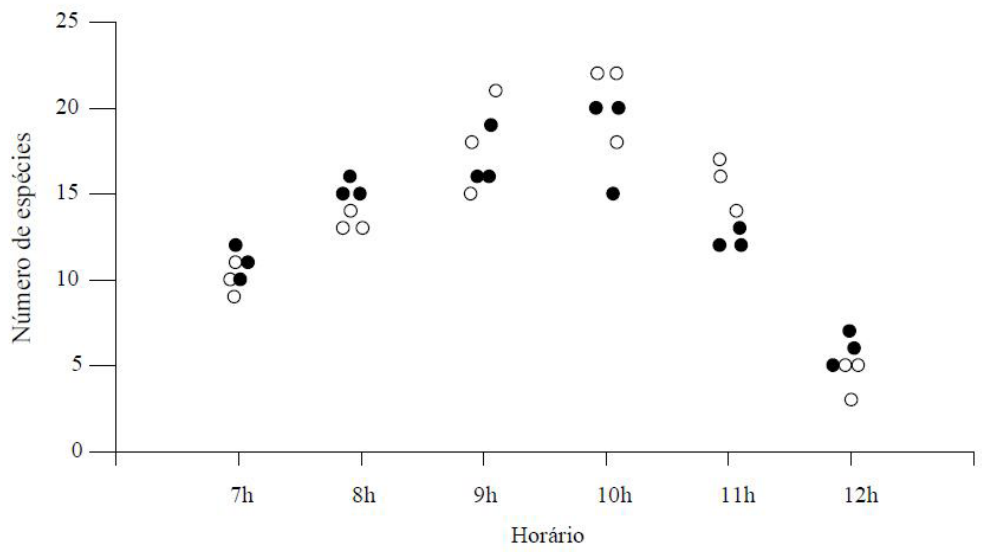

Fig. 1 - Número de espécies de insetos visitantes florais em algodão Bt (pontos preenchidos) e não-Bt (pontos vazios). Maracaju, MS, 2008.

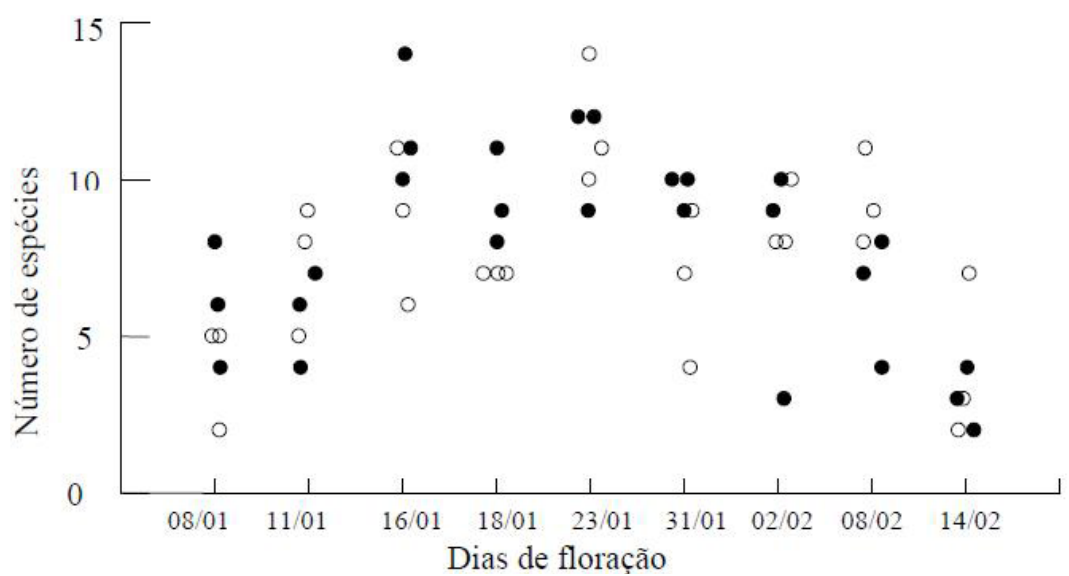

Fig. 2 - Número de espécies de visitantes florais em algodoeiro Bt (pontos preenchidos) e não-Bt (pontos vazios) durante o período de floração. Maracaju, MS, 2008.

Tabela 2 - Número médio de espécies ( \pm desvio padrão) de insetos visitante florais em seis classes de horários, durante o período de floração do algodoeiro Bt e não-Bt. Marcaju, MS, 2008.

\begin{tabular}{lccc}
\hline \multirow{2}{*}{ Horário } & \multicolumn{2}{c}{ Algodão } & Total \\
\cline { 2 - 3 } & $\mathrm{Bt}$ & $10,00 \pm 1,00 \mathrm{fh}$ & $10,50 \pm 1,05 \mathrm{~A}$ \\
$7 \mathrm{~h}-8 \mathrm{~h}$ & $11,00 \pm 1,00 \mathrm{ef}$ & $13,33 \pm 0,58 \mathrm{cf}$ & $14,33 \pm 1,21 \mathrm{~B}$ \\
$9 \mathrm{~h}-9 \mathrm{~h}-10 \mathrm{~h}$ & $15,33 \pm 0,58 \mathrm{~b}$ & $18,00 \pm 3,00 \mathrm{abc}$ & $17,50 \pm 2,26 \mathrm{C}$ \\
$10 \mathrm{~h}-11 \mathrm{~h}$ & $17,00 \pm 1,73 \mathrm{abcd}$ & $20,67 \pm 2,31 \mathrm{a}$ & $19,50 \pm 2,67 \mathrm{C}$ \\
$11 \mathrm{~h}-12 \mathrm{~h}$ & $18,33 \pm 2,89 \mathrm{ab}$ & $15,67 \pm 1,58 \mathrm{bcde}$ & $14,00 \pm 2,10 \mathrm{~B}$ \\
$12 \mathrm{~h}-13 \mathrm{~h}$ & $12,33 \pm 0,58 \mathrm{def}$ & $4,33 \pm 1,16 \mathrm{~g}$ & $5,17 \pm 1,33 \mathrm{D}$ \\
\hline Total & $6,00 \pm 1,00 \mathrm{gh}$ & $13,67 \pm 5,72$ & \\
\hline
\end{tabular}

Valores seguidos pela mesma letra minúscula não diferem estatisticamente e seguidos de mesma letra maiúscula não diferem (teste de comparações múltiplas de Tukey, $a=0,05$ ). 
Por outro lado, a riqueza de espécies não variou entre os cultivares de algodão ( $\mathrm{F}=0,300 ; \mathrm{gl}=1 ; \mathrm{p}=$ $0,587)$ e nem entre as classes da interação ( $F=1,980 ; \mathrm{gl}$ $=8 ; \mathrm{p}=0,078)$, todavia, variou entre os dias defloração ( $\mathrm{F}=8,776 ; \mathrm{gl}=8 ; \mathrm{p}<0,001)$ onde no início e no final da floração constatou-se a menor riqueza de espécies independentemente do cultivar de algodão (Fig. 2).

Conforme o ciclo fenológico do algodoeiro e o período de abertura das flores na área amostral, os visitantes florais apresentaram variação no total de indivíduos coletados durante o período das amostragens atingindo um número elevado de indivíduos entre a fase da floração plena e o final da floração. Os insetos visitaram as flores quando as plantas tinham entre 75 e 85 dias após a emergência, fase de maior emergência de botões e flores. Conforme foram diminuindo os botões florais, consequentemente diminuíram os visitantes.
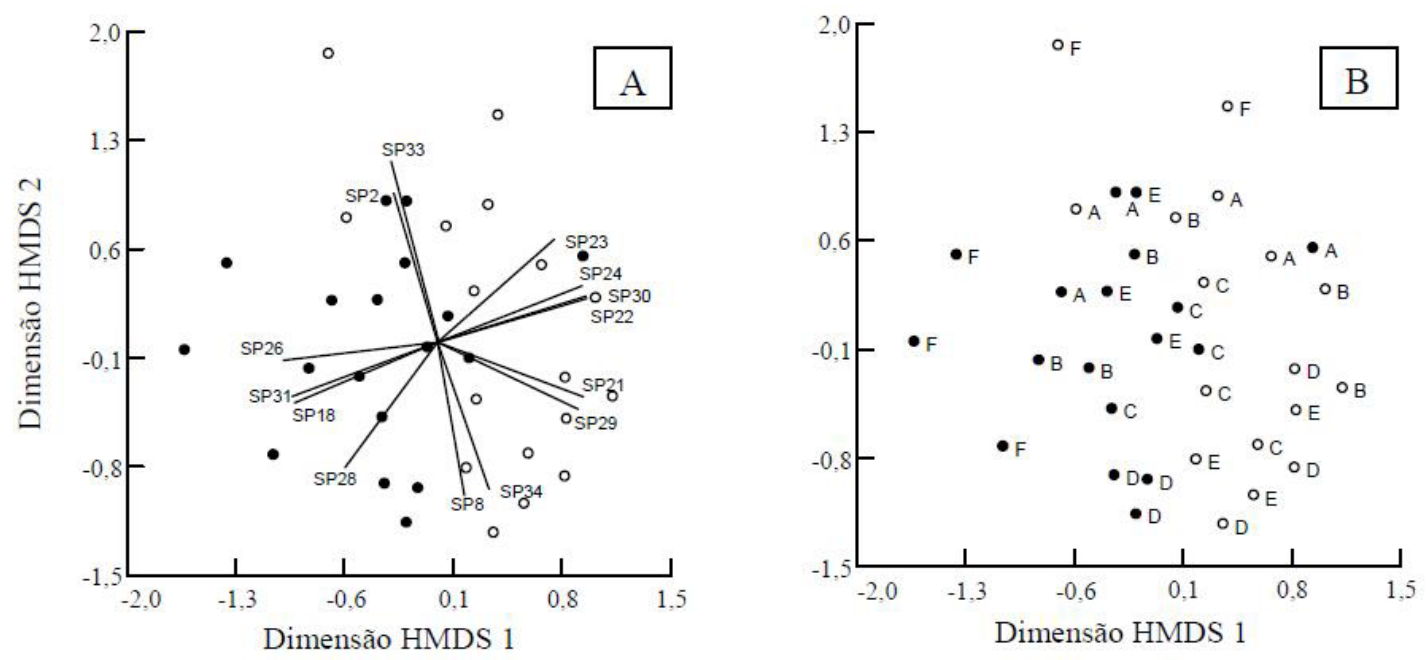

Fig. 3 - Ordenação por HMDS em duas dimensões (stress = 0,26) dos horários de visitação em flores de algodão Bt (pontos preenchidos) e não-Bt (pontos vazios). No gráfico A, os vetores indicam a contribuição relativa de cada espécie de visitante floral para a separação dos horários no plano da ordenação ( $\mathrm{r}>0,4)$ : SP2- O. antipathus, SP8- B. (fervidobombus) morio, SP33- H. convergens, SP23- C. malleatus, SP24- Ichneumonidae Gênero A sp1, SP30- A. exotica, SP22- A. argilacea, SP21- S. (Scymnus) sp., SP29- H. virescens, SP34- A. mellifera, SP28- Diabrotica sp., SP18- E. ello, SP31- C. sanguinea, SP26- C. joliveti. No gráfico B, as letras representam os horários de visitas no mesmo plano da ordenação (A - 7h às $8 \mathrm{~h}$, $B-8 h$ às $9 h, C-9 h$ às $10 h, D-10 h$ às $11 h, E-11 h$ às $12 h$ e $F-12 h$ às $13 h$ ).
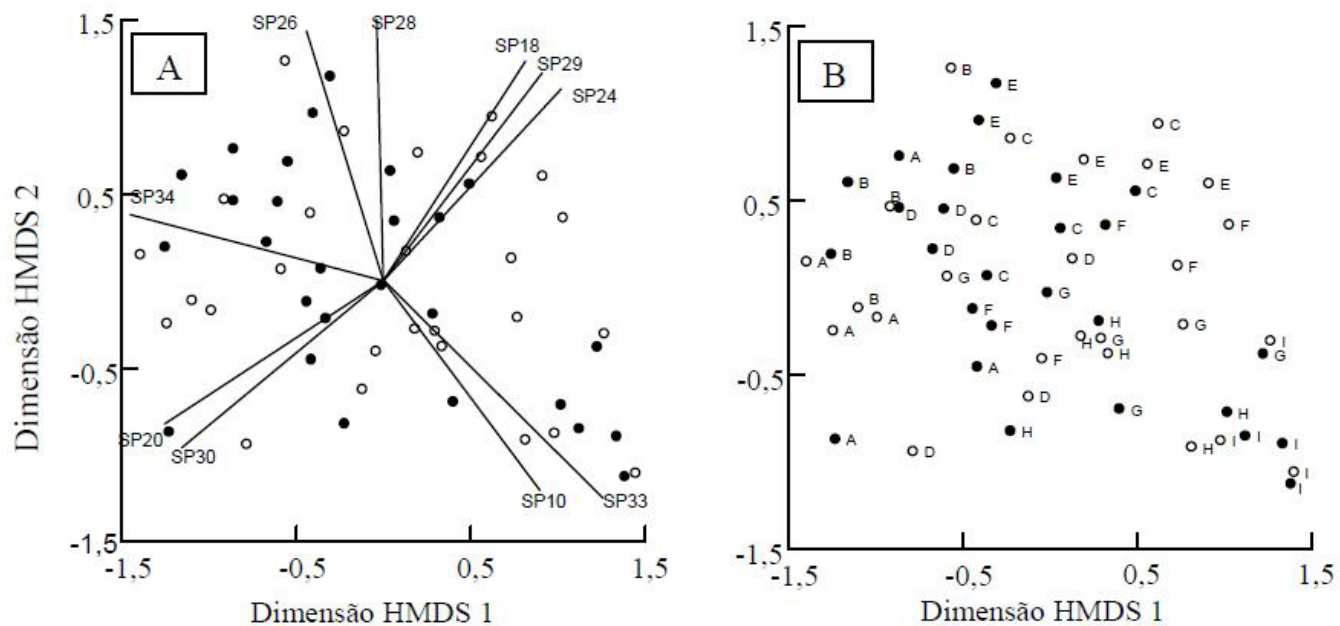

Fig. 4- Ordenação por escalonamento multidimensional híbrido (HMDS, stress =0,24) dos dias de floração de algodoeiro $\mathrm{Bt}$ (pontos preenchidos) e não-Bt (pontos vazios). No gráfico $\mathrm{A}$, os vetores indicam o quanto cada espécie contribuiu para a separação de dias de floração. SP10 - Tachinidae sp.2, SP33 - H. convergens, SP30 - A. exotica, SP20 - Pyralidae sp. , SP34 - A. mellifera, SP26 - C. joliveti, SP28 - Diabrotica sp., SP18 - E. ello, SP29- H. virescens, SP - 24 Ichneumonidae Gênero A sp.1. No gráfico B as letras representam os dias de coletas no mesmo plano da ordenação (A - 8/jan/2008, B -11/jan/2008, C -16/jan/2008, D - 18/jan/2008, E -23/jan/2008, F - 31/jan/2008, G - 2/fev/2008, H - 8/fev/2008, I - 14/fev/2008). 
Além de apresentar diferença na riqueza de espécies, a composição dessas espécies foi diferente no decorrer da manhã. Houve variação entre os horários de visitação (Pillai-Trace $=1,02 ; \mathrm{F}=4,77 ; \mathrm{gl}=10$ e 46; $\mathrm{p}<0,001$ ), entre os tipos de algodão (Pillai-Trace $=$ 0,$56 ; \mathrm{F}=14,01 ; \mathrm{gl}=2$ e 22; $\mathrm{p}<0,001$ ) e também da interação entre horários e tipo de algodão (PillaiTrace $=0,80 ; \mathrm{F}=3,09 ; \mathrm{gl}=10$ e 46; $\mathrm{p}=0,004$ ) (Fig. $3)$. E essa diversidade de espécies foi diferente tanto entre horários como entre tipo de algodão, ou seja, a espécies que visitavamo algodão Bt não eram as mesmas que visitavam o não-Bt naquele mesmo horário. Por exemplo, abelhas da espécie $B$. (fervidobombus) morio e os coleópteros $C$. joliveti e $C$. sanguinea foram coletados mais frequentemente no cultivar Bt e das $8 \mathrm{~h}$ às $10 \mathrm{~h}$, e entre as $10 \mathrm{~h}$ e $12 \mathrm{~h}$ a abelha $A$. mellifera ocorreu principalmente no algodão não-Bt.

As espécies que mais contribuíram para diferenciar as amostras, em ordem decrescente, foram Apis mellifera, $H$. convergens, $P$. includens, $C$. sanguinea, $A$. exotica, $H$. virescens, Diabrotica sp., A. gemmatalis, C. joliveti, E. connexa, Ichneumonidae Gênero A sp., C. malleatus, A. argilacea, Scymnus (Scymnus) sp., Pyralidae sp., Macrodactylus sp., E. ello, A. grandis, Tachinidae sp.4, Bombylidae Gênero A sp., E. agrotina, $S$. frugiperda, $D$. lineare, Tachinidae sp.2, $O$. gastrotactus, Bombus (Fervidobombus) morio, Trigona spinipes, Odontochila sp., H. festiva, Tachinidae sp.3, Stratiomidae Gênero A sp.1, O. antipathus e Oxaea flavescens.

A riqueza de espécies também variou conforme os dias de floração, para ambos os tratamentos (Pillai-Trace $=1,41 ; \mathrm{F}=10,76 ; \mathrm{gl}=16$ e 72; $\mathrm{p}<0,001$ ), mas não entre os cultivares de algodão (Pillai-Trace $=0,046 ; \mathrm{F}=0,80 ; \mathrm{gl}=2$ e 35; $\mathrm{p}=0,460)$, e nem entre as classes da interação entre os dias e o tipo de algodão (Pillai-Trace $=0,490 ; \mathrm{F}=1,47 ; \mathrm{gl}=16$ e 72; $\mathrm{p}=0,138$ ) (Fig. 4).

Algumas das espécies contribuíram decisivamente para a diferença entre os dois tratamentos avaliados. Por exemplo, Ichneumonidae Gênero A sp.1 contribuiu para a diferenciação na composição das espécies de visitantes florais do algodão não$\mathrm{Bt}$, principalmente entre os dias 23 e 31 de janeiro, período de maior visita, Tachinidae sp. 2 apresentou maior significância nos dias 2 de fevereiro na cultura de algodão Bt e em 8 de fevereiro no não-Bt. Já o coleóptero $H$. convergens foi significante na composição das espécies nos dois tipos de algodão, e sua visitação foi mais frequente no final do período da floração, que ocorreu em meados de fevereiro. A espécie Diabrotica sp. visitou as flores com maior frequência em torno do dia 23 de janeiro, tanto no algodão Bt como no não-Bt. A ocorrência de C. joliveti foi significativa no algodão não-Bt no começo da floração, em torno do dia 11 de janeiro, e no algodão $\mathrm{Bt}$ em torno do dia 23 de janeiro. Já a abelha A. mellifera teve significância em todo o período da floração para os dois cultivares de algodão.

No início e no final do período de florescimento o número de visitantes florais foi inferior, o que pode ser explicado pela menor oferta de flores. $\mathrm{O}$ menor número de insetos, principalmente abelhas, foi coletado no primeiro horário de amostragem, e também no começo de janeiro, quando foram registradas as menores temperaturas ocorridas no período de avaliação. Outro fato observado foi a diminuição de insetos em torno dos 12 dias de fevereiro, época em que a precipitação foi mais acentuada na região, com dias contínuos de chuva.

De acordo com os resultados desta pesquisa, o algodoeiro Bt apresentou maior quantidade de insetos visitantes florais, mas a gama de espécies que visitaram o algodoeiro não-Bt foi mais diversa. E, também, a composição de espécies que visitou as flores do algodoeiro Bt e algodoeiro não-Bt é diferente, essa diferença pode estar relacionada com um efeito indireto da planta Bt sobre estes insetos não-alvo. Por exemplo, no algodoeiro não-Bt houve maior presença de pragas, esse fator pode ter influenciado a presença de algumas espécies e a ausência de outras; já o algodoeiro Bt expressa toxina proveniente do $B$. thuringiensis o que talvez possa ter influenciado a ausência de algumas espécies. Portanto, sugerimos outros estudos sobre composição de visitantes florais do algodoeiro que compare cultivar GM e sua isolinha, em quatro ou mais áreas de produção, e em pelo menos três anos agrícolas e, assim, confirmar o efeito do algodão Bt sobre estes insetos benéficos.

\section{AGRADECIMENTOS}

Agradecemos aos taxonomistas da UFPR: Dr. Mário Antonio Navarro da Silva, Dra. Lucia Massutti de Almeida, Dr. Germano Henrique Rosado-Neto, Dra. Maria Christina de Almeida, à Mirian Morales Nunes, ao Geovan Henrique Correa, Paschoal Coelho Grossi e Bolívar Rafael Garcete Barret. Ao Dr. Alexandre Specht da Universidade de Caxias do Sul. E também a todos que colaboraram com o trabalho de campo.

\section{REFERÊNCIAS}

ANACLETO, D.A. de; MARCHINI, L.C. Análise faunística de abelhas (Hymenoptera, Apoidea) coletadas no cerrado do Estado de São Paulo. Acta Scientiarum. Biological Sciences, v.27, n.3, p.277-284, 2005.

ANDOW, D. The risk of resistance evolution in insects of transgenic insecticidal crops. Collection of Biosafety Reviews, v.4, p.142-199, 2008. 
ARPAIA, S.; FONSECA, V.L.I.; PIRES, C.S.; SILVEIRA, F.A. Non-target and biodiversity impacts on pollinators and flower-visiting insects. In: HILBECK, A.; ANDOW, D.; FONTES, E. (Ed.). Environmental risk assessment of genetically modified organisms: a case study of Bt cotton in Brazil. Wallingford: CABI, 2006. 373p. Chap. 7.

BETZ, F.S.; HAMMOND, B.G.; FUCHS, R.L. Safety and advantages of Bacillus thuringiensis-Protected plants to control insect pest. Regulatory, Toxicology and Pharmacology, v.32, p.156-173, 2000.

BIRCH, R.G. Plant transformation: Problems and Strategies for Practical Application. Annual Review Plant Physiology and Plant Molecular Biology, v.48, p.297-326, 1997.

BRAY, J. R.; CURTIS, J. T.. An ordination of the upland Forest communities of southern Wisconsin. Ecological Monographs, v. 27, p. 325-349, 1957.

COMISSÃO TÉCNICA NACIONAL DE BIOSSEGURANÇA. (Brasil) 2008. Disponível em: <http://www. ctnbio.gov.br/index.php/content/view/12673.html>. Acesso em: nov. 2008.

COMISSÃO TÉCNICA NACIONAL DE BIOSSEGURANÇA. 2009. (Brasil). Disponível em: <http://www. ctnbio.gov.br/index.php/content/view/12750.html>. Acesso em: nov. 2010.

FAITH, D.P.; MINCHIN, P.R.; BELBIN, L. Compositional dissimilarity as a robust measure of ecological distance. Vegetatio, v. 69, p.57-68, 1987.

GRAVENA, S. O controle biológico na cultura algodoeira. Informe Agropecuário, v.9, p.3-15, 1983.

HONG, L.T.T.; TÂM, D.Q.; TUÂN, H.A.; et al. Potencial effects of transgenic Cotton on flower visitors in Vietnam. In: ANDOW, D.A.; HILLBECK, A.; VAN TUAT, A.N. (Ed.). Environmental Risk Assessment of Genetically Modified Organisms: challenges and opportunities with Bt cotton in Vietnam. Wallingford: CABI, 2008. 432p. Chap. 9.
JAMES, C. Global Status of Commercialized Biotech/GM Crops: 2011. Ithaca, NY: ISAAA, 2011. (ISAAA Brief oㅡ 43).

MONNERAT, R.G.; BRAVO, A. Proteínas bioinseticidas produzidas pela bactéria Bacillus thuringiensis: modo de ação e resistência. In: MELO, I.S.; AZEVEDO, J.L. (Ed.). Controle biológico 3. Jaguariúna: Embrapa Meio Ambiente, 2000. p.163-200.

PIRES, C.; SILVEIRA, F.A.; PEREIRA, F.O.; PAES, J.S. de; SUJII, E.R.; FONTES E. Protocolo de amostragem de visitantes florais em algodoeiro (Gossypium spp.). Brasília: Embrapa Recursos Genéticos e Biotecnologia, 2006a. (Boletim de Pesquisa e desenvolvimento 131).

PIRES C.; SILVEIRA F. da; CARDOSO C.F.; OLIVEIRA, G.M. de; PEREITA, F.F.O.; SOUZA, V.V. de; NAKASU, E.Y.T.; PAES, J.S.O.; TELES, É.; SILVIE, P.; RODRIGUES, S.; MIRANDA, J.; SCOMPARINI, J.; BASTOS, C.; OLIVEIRA, G.S.; OLIVEIRA, J.E.; SANTOS, J.B.; BARROSO, P.A.V.; SUJII, E.; FONTES, E. Visitantes florais em espécies cultivadas e não cultivadas de algodoeiro (Gossypium spp.), em diferentes regiões do Brasil. Brasília: Embrapa Recursos Genéticos e Biotecnologia, 2006b. 40p. (Boletim de pesquisa e desenvolvimento, 148).

SANCHEZ-JUNIOR, J.L.B.; MALERBO-SOUZA, D.T. Frequência dos insetos na polinização e produção de algodão. Acta Scientiarum Agronomy, v.26, n.4, p.461-465, 2004.

SHANNON, C.E.; WEAVER, W. The mathematical theory of communication. Urbana: University of Illinois Press, 1949.

TELLES, E. Inventário dos visitantes florais da cultura do algodão na região de Rondonópolis. Monografia (Curso Agronomia) - Faculdades Integradas de Rondonópolis Agronomia. Rondonópolis, 2006.

THAPA, R.B. Honeybees and other insect pollinators of cultivated plants: A review. Journal of the Institute of Agriculture and Animal Sciences, v.27, p.1-23, 2006.

Recebido em 9/3/11

Aceito em 26/5/12 